\title{
Using Behavioral Knowledge for Situated Prediction of Movements
}

\author{
Michael Arens, Artur Ottlik, and Hans-Hellmut Nagel \\ Institut für Algorithmen und Kognitive Systeme, \\ Fakultät für Informatik der Universität Karlsruhe (TH), \\ 76128 Karlsruhe, Germany \\ \{arens|ottlik|nagel\}@iaks.uni-karlsruhe.de
}

\begin{abstract}
The textual description of video sequences exploits conceptual knowledge about the behavior of depicted agents. An explicit representation of such behavioral knowledge facilitates not only the textual description of video evaluation results, but can also be used for the inverse task of generating synthetic image sequences from textual descriptions of dynamic scenes. Moreover, it is shown here that the behavioral knowledge representation within a cognitive vision system can be exploited even for prediction of movements of visible agents, thereby improving the overall performance of a cognitive vision system.
\end{abstract}

\section{Introduction}

Artificial cognitive vision systems are supposed to analyze single video frames or entire video sequences in order to construct a conceptual representation of the depicted scene $[12,7]$. Based on such a conceptual scene description, natural language descriptions can be derived $[15,19,3,6]$.

It has been shown that encompassing natural language descriptions of image sequences from innercity intersections can be generated by employing explicitly formulated knowledge about the discourse in question [11,6]. Especially the explicit representation of behavioral knowledge in form of so-called situation graph trees (SGTs) enables the cognitive vision system to aggregate conceptual primitives derived from quantitative vision results to complex, context-dependent concepts (compare $[11,1]$ ).

In $[19,3]$ the authors pointed out that by solving the inverse task of creating a synthetic video sequence from textual descpritions of a time-varying scene (see, too, [4]), the performance of the overall system can be improved: the generated text is analyzed in order to re-create the initial scene which led to the given textual description. Supposing that original and synthetic image sequence can be compared, significant differences between original and synthetic image sequence can point to details which need to be incorporated into the textual description of the initial scene. The differences are analyzed in order to come up with a better textual description, i. e., one that minimizes the differences between the original sequence and the synthetic sequence which one might create as a 'mental image' when reading the textual description. 
The authors of [20] use conceptual knowledge employed within a surveillance system to create test sequences for their vision system. This usage of background knowledge normally employed within a vision system is an elegant way to test the completeness of the overall system in two senses: can every (visual) behavior, which the surveillance system is supposed to detect, be modelled by this knowledge ? And, moreover, is every behavior modelled within the knowledge base also detectable by the vision system ?

In [9], still another use of conceptual knowledge employed in a vision context has been presented: behavioral knowledge in form of situation graph trees (SGTs) was not only used to link quantitative vision results with complex concepts in order to generate textual descriptions of a scene. In addition to this, the knowledge represented as SGTs was employed to generate quantitative predictions of where an agent recognized by the vision system would be positioned in the future, e.g., in the next frame of the video sequence.

This leaves the conceptual knowledge employed within a cognitive vision system with (at least) three main purposes it should be solving or enabling: first, the most obvious purpose of behavioral knowledge within a vision context is to link quantitative vision results to complex concepts. Second, the same knowledge should be usable to generate synthetic video sequences from conceptual or textual descriptions of scenes. And last, the behavioral knowledge represented within the vision system should be capable of predicting the behavior of agents not only in a conceptual, but also in a quantitatively exploitable way.

In the sequel, we will show that, in principle, SGTs as a representational form for behavioral knowledge satisfy all three demands mentioned above: in Section 2 we will first briefly recapitulate SGTs as such and how they can be of benefit in conceptualizing video sequences of, e.g., innercity road traffic. Section 3 will be concerned with the generation of synthetic video sequences based on SGTs. In the present contribution, we will extend the work presented in [1] by showing how conceptual representations of a certain behavior can be linked to quantitative movements. Section 4 will then demonstrate how this linkage can also be used for the situated prediction of movements within the quantitative processes implementing computer vision approaches towards vehicle tracking in real-world image sequences.

\section{Cognitive Vision}

XTRACK (compare $[10,13])$ is a model-based vision system capable of detecting and tracking vehicles in image sequences of innercity road traffic. Model-based in this context means that the system comprises explicit representations of several pieces of background knowledge. These include geometric models of vehicles and other scene components like lane models. Additionally, an algebraic vehicle motion model is used within a Kalman filter approach $[5,8]$ to describe the systematic changes which vehicle poses undergo during vehicle motion. Such a pose is called the state of a vehicle and is to be estimated by XTRACK for each recognized vehicle for each video frame. As a result of the tracking process 
conducted by XTRACK, the system derives a geometric scene description (GSD) [17] for each image frame, comprising both the static scene components and the time-varying states of recognized vehicles.

The GSD is imported into a conceptual inference system based on the fuzzy metric temporal Horn logic (FMTHL) introduced by [18], which facilitates logic inference on time-varying and fuzzy facts and rules. Based on the state vectors and other scene descriptions imported as facts into this inference system, the system uses additional background knowledge - this time in form of logic rules to derive conceptual primitives from the imported facts. This knowledge is called the terminology and defines, e.g., which velocity of a vehicle should be labeled with the concept fast or which constellation of positions of two vehicles should be denoted as in_front_of. Note that both terms are vague and thus are modelled as fuzzy predicates within FMTHL [7].

Defining more complex concepts in terms of a terminology quickly becomes infeasible, because the same value (or change in values) of vehicle states are combined with the same conceptual primitives all the time. No difference is made by terminologic rules between, e.g., a vehicle changing_the_lane while starting to overtake another car and changing_the_lane after finishing the process of overtaking. We employ situation graph trees (SGTs) in order to express such more complicated dependencies of concepts on the context in which a certain value (change) should be labeled with that concept.

SGTs are graphs whose nodes are given by situation schemes. A situation scheme combines the description of the state of an agent (e.g., a vehicle) in terms of conceptual primitives with the description of the actions this agent might perform whenever it is in that situation. Situation schemes build situation graphs by connecting single schemes by so-called prediction edges. These directed edges define a temporal successor relation on situation schemes, i.e., whenever a scheme was instantiated by a vehicle, all those schemes describe plausible successor situations which can be reached from the present scheme by following a prediction edge. A second type of directed edges - called particularization edges - connect situation schemes with situation graphs. These edges represent the fact that a single situation can be described in more detail by a whole sequence of other situations. Thus, particularization edges connect situation schemes with those graphs comprising such detailing sequences. In summary, an SGT describes the state of an agent as it is embedded into the context of past and possible future states and possible actions of this agent together with more detailed or more general possible descriptions (compare, e.g., [1]).

The question which vehicle recognized by XTRACK instantiates which situation of the employed SGT can be answered by traversing the SGT along prediction- and particularization edges. SGTs can therefore be transformed into logic programs within FMTHL (compare [18]). Thus, they perfectly fit to the conceptual primitives derived in our conceptual inference system. Such an SGTtraversal results in a conceptual scene description (CSD) comprising complex concepts, e.g., - to stay with the example mentioned above - starting_to_overtake, finished_overtaking, and overtaking in general. These concepts can then be further 
transformed by a natural language generation system into natural language text. For details on this transformation see [6].

\section{Controlled Imagery Generation}

In [16] (see also [14]) it has been described how conceptual primitives - as derived by the inference subsystem of a cognitive vision system - can be (re-)visualized. In [2] first results have been presented on how such a visualization can be performed on natural language input texts, again, using texts previously produced by a combination of a vision system together with an inference- and a natural language sub-system. However, this approach did not exploit exactly the same knowledge (i.e., the SGTs) which was originally employed to produce the texts. Such a procedure is, however, a prerequisite to test the knowledge base for completeness as has been motivated in Section 1.

SGTs can be used to solve certain problems arising due to the inherent incompleteness of natural language texts [1]: because these texts are normally aimed at human readers, these readers are assumed to be able to use their commonsense knowledge in order (i) to embed given information into a plausible context and (ii) to detail general information to a degree sufficient for imagining what is going on in the scene decribed by the text.

For both tasks, context-embedding and information-detailing, an algorithm based on the structure of SGTs and the behavioral knowledge stored therein has been presented in [1]. Given a sequence of situations, e.g., derived from a natural language input text, the algorithm returned so-called maximized SGTcompatible behaviors. These behaviors comprised the information given by the initial situation sequences, but described in the most detailed way possible based on the knowledge represented by the SGT and embedded into a context also compatible with that SGT (for details, see [1]). What remained to be done at that point was to transform such an SGT-compatible behavior - a situation sequence itself - into an actual video sequence visualizing this behavior.

It turned out that the visualization of situation sequences can be facilitated by defining suitable action schemes within situation schemes. The connection between these action schemes and the states of vehicles in a scene to be visualized is established by the motion model already defined within the vision system XTRACK. This motion model had to be extended, however.

\subsection{From Action Predicates to Vehicle States}

The action scheme of a situation scheme defines actions to be performed either by the vision system itself or by the agent vehicle in question in terms of action predicates. The vision system might, e.g., print out a string announcing the corresponding situation scheme to be instantiated. The vehicle itself might be expected, e.g., to follow_the_lane. In the descriptive system approach sketched in Section 2, however, the action scheme was exclusively used for the former purpose. The latter can, however, be facilitated by defining complex action predicates in terms of a so-called action terminology. 
An action terminology describes each complex action predicate in terms of more simple actions. Basic actions or action-primitives in the case of vehicles are acceleration ('longitudinal control') and steering commands ('lateral control'). The action terminology can - as the terminology which defined complex concepts on conceptual primitives - be expressed in form of logic rules within FMTHL. Thus, the background knowledge accessible during the vision task described in Section 2 can also be used within this action terminology. For example, an action predicate like follow_lane - which should cause a vehicle to stay on the lane it is presently driving on - can use the geometric information stored about the lane structure of a road scene together with the present position and speed of the vehicle in question to break down this action predicate into steering commands. These steering commands together with commands concerning the acceleration of an agent result in desired values for the speed and heading of each vehicle to be visualized. By feeding these desired values for speed and heading of vehicles into a controllable vehicle motion model (see Appendix A), a sequence of situations results in a sequence of vehicle states compatible with the situation sequence - the behavior. The resulting vehicle states can then be visualized by using standard graphic algorithms.

\section{$3.2 \quad$ Results}

Based on an SGT describing the (expected) possible behavior of vehicles at an intersection, we selected one situation scheme to be comprised in the behaviors we want to visualize, namely driving_towards_intersection. Notice that this selection process might be initialized by the analysis of a natural language text as suggested in [2] or, as presented here, by interactively selecting a scheme directly from within an SGT in order to view all behaviors compatible with this scheme.

Using the algorithm proposed in [1], we derived all maximized SGT-compatible behaviors with respect to the given SGT and the selected scheme. These behaviors themselves are sequences of situation schemes, as can examplarily be seen in Figure 1. Notice that the depicted behavior is only one of six behaviors which have been created, reflecting the fact that more than one behavior represented by the SGT is conceptually compatible with the situation scheme initially selected: several created behaviors comprise the notion of an additional vehicle, i.e., a vehicle to which the agent (whose behavior is modelled) would have to give way while crossing the intersection. From the six behaviors compatible with the selected scheme, we manually selected one to be visualized.

Each situation scheme comprised in that behavior contains action predicates. These are (i) follow_lane(Agent), which causes the agent to stay on the lane it is presently driving on, (ii) follow_lseg(Agent) by which the agent is caused to stay on the centerline of the present segment of the lane he is driving on, and (iii) accelerate_to(Agent,normal), which results in the agent accelerating to a velocity which is given by the conceptual value of normal. These action predicates are translated into action-primitives on the basis of the action terminology: the definition of follow_lane takes the present position of the agent and additional knowledge about the lane structure into account to result in the 


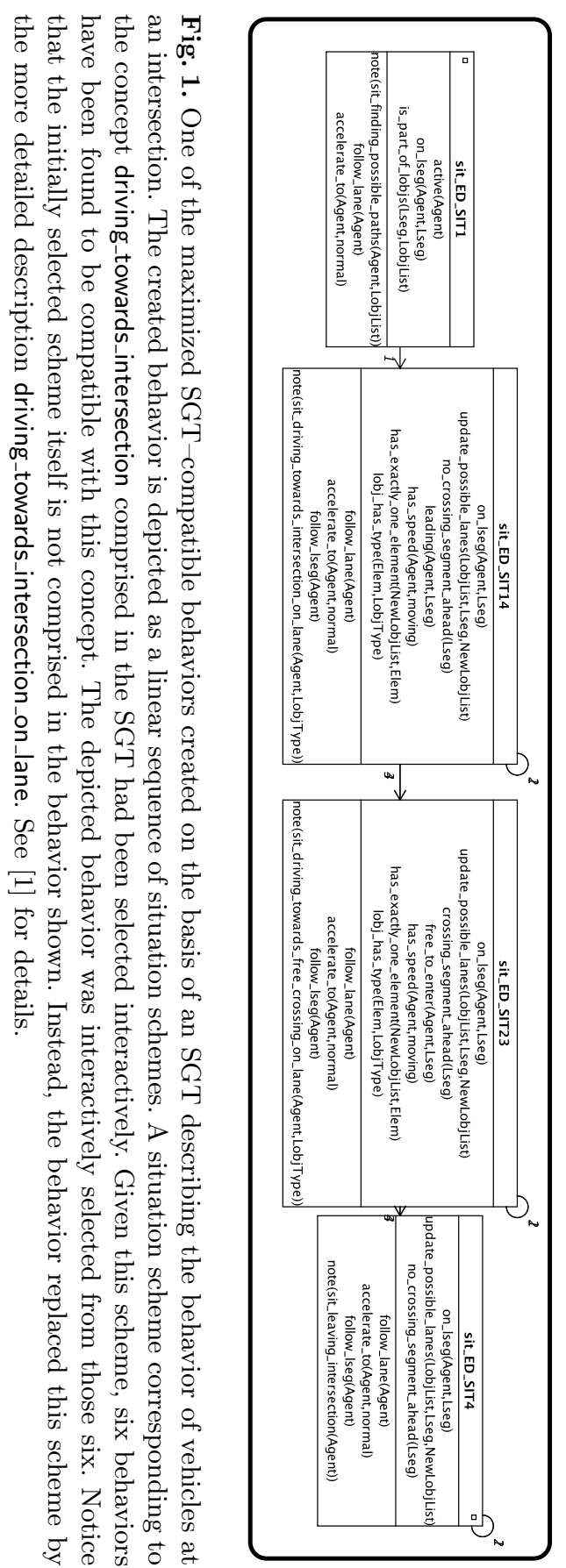


action-primitive steer_to(Agent, <num $>$ ) where $<$ num $>$ denotes the absolute numerical value for the resulting steering angle. In the same way, follow_lseg results in the action-primitive steer (Agent, $<$ num $>)$. Here, $<$ num $>$ denotes a relative steering angle the agent vehicle is supposed to employ. The definition of the predicate accelerate_to translates the conceptual value normal into a numerical value <num> for the velocity the agent vehicle is supposed to reach. This results in the actionprimitive accelerate_to(Agent, $<$ num $>$ ). These action-primitives are used to control the agent vehicle motion model described in Appendix A.

By manually selecting an initial vehicle state, the created behavior can be traversed like a normal SGT: the initial vehicle state leads to the instantiation of the first situation scheme within the behavior. The action-primitives resulting from this scheme create a new vehicle state based on the controllable motion model. This state again leads to the instantiation of the same or the next scheme in the behavior, and so on. The trajectory, i.e. the sequence of vehicle states, created from the behavior selected from Figure 1 is depicted in Figure 2. Notice that the created image sequence used a single image frame from a video sequence of an intersection as background. The trajectory data was superimposed to this image, but could also have been used to create any other view on the 3Dscene represented by the trajectory data created from within the SGT and the geometric background knowledge about the static scene components. For one such different view see, too, Figure 2.

\section{Cognitive Vision with Situated Prediction of Movements}

As has been shown in the preceding section, each situation scheme comprised in a maximized SGT-compatible behavior contained action predicates which could be translated into action-primitives. These primitives were executed by a motion model, resulting in time-varying state vectors of agent vehicles.

Obviously, the action schemes defined in situation schemes are not only accessible via the creation of maximized SGT-compatible behaviors, but via the SGT-traversal performed during the vision task, too. How these action schemes can be used in the quantitative vision process will be described in the sequel.

Remember that XTRACK employs a Kalman filter approach to estimate the state of vehicles to be tracked. This approach relies on a measurement at each video frame, but also on a prediction of the state to be expected in that frame. This prediction is generated with the help of a motion model accessible to XTRACK. This motion model normally assumes - due to the lack of better assumptions - a constant steering angle and constant speed of observed vehicles. This - obviously not correct - assumption can be refined by the knowledge represented in SGTs.

Once XTRACK has estimated the state of a vehicle and the conceptual subsystem has instantiated a situation scheme for that vehicle, action predicates defined in that scheme can be executed employing the controllable motion model described in Appendix A. This leads to an updated state vector of that vehicle. 


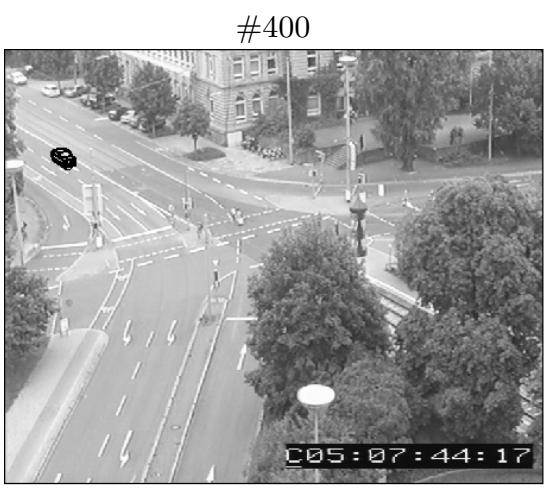

\#600

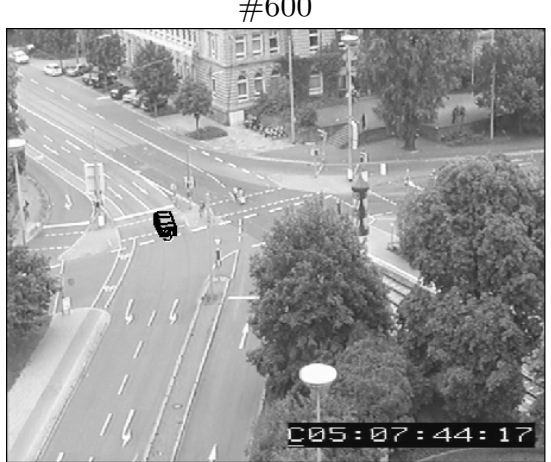

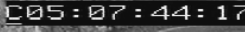

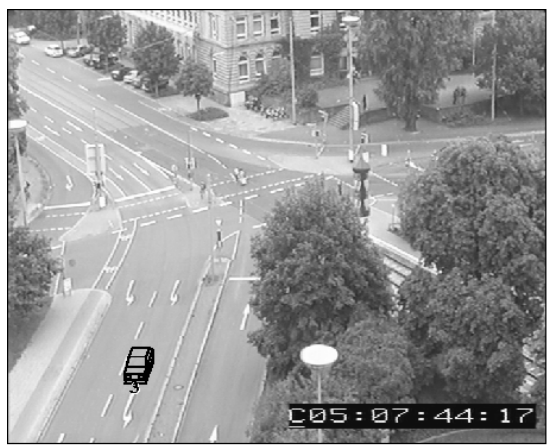

\#800
\#500

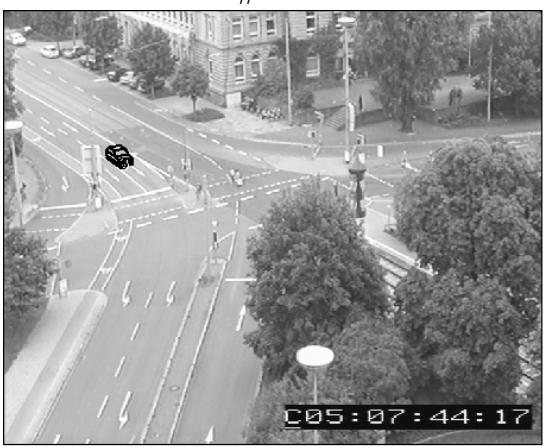

\#700
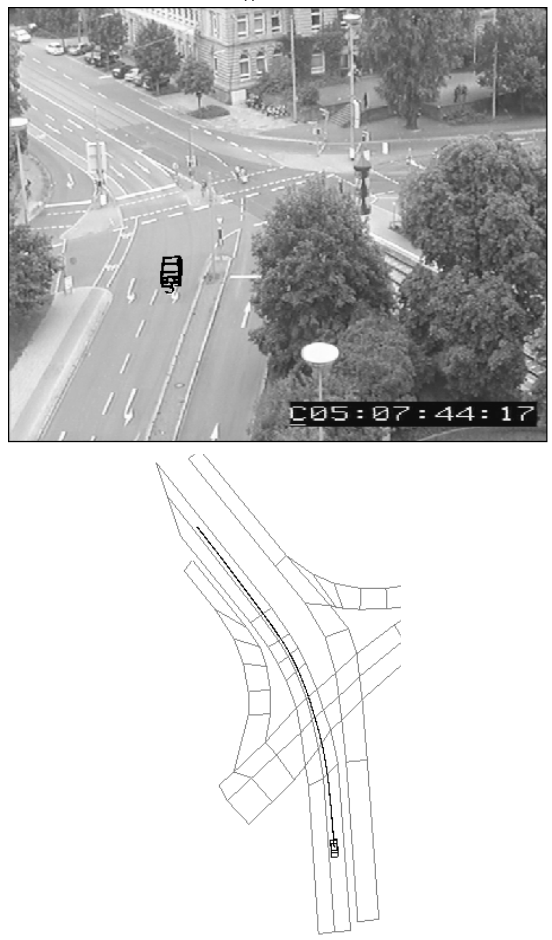

birds eye view

Fig. 2. Representative frames of the visualization of the behavior given in Figure 1. All agent vehicle states have been superimposed to a single frame of an intersection video sequence. The bottom-right figure shows a birds eye view onto the lane model with the synthetically created vehicle trajectory superimposed. 
By using this state vector as a prediction within the Kalman filter, XTRACK can profit from the knowledge about the expected behavior of vehicles concerning steering and acceleration.

SGTs have been used already to predict the movement of agents [9]. This was only done, however, under certain circumstances (at least $80 \%$ of occlusion of a vehicle due to static scene components) and for a few selected situations. In addition to these restrictions, the actions to be performed by a simulated agent i.e., the internal representation of the vehicle recognized in the scene - were tied closely to situations, i.e., one situation corresponded to one action implemented directly as a procedure of the core vision system. The approach described here instead defines complex actions in terms of an action terminology. This leads to action-primitives which always have the same form (acceleration and steering commands). The overall conceptual sub-system, therefore, is much more flexible with respect to extensions: new situations can define their action scheme in terms of the action terminology, while the motion model controlled by the resulting action-primitives remains unchanged once it has been fully implemented.

\subsection{Results}

Figure 3 shows tracking results obtained with XTRACK on a video sequence of an innercity intersection. For this experiment, four vehicles were initialized interactively and then tracked by XTRACK automatically: The left column of Figure 3 show the results obtained using the original motion model which assumes a constant steering angle and velocity of vehicles. This assumption is accurate enough for XTRACK to track vehicles under optimal measurement condition, i.e., when the vehicles are not occluded by other scene components. Under such conditions, the vision system can adapt to subtle changes in steering angle or velocity simply during the updating step of the prediction/update cycle realized by the Kalman filter. In the sequence depicted in Figure 3, however, all four vehicles become partially or totally occluded by a tree and change both their steering angle and velocity during this period. As a consequence, two of the four vehicles cannot be tracked correctly and get lost.

The right column depicts results on the same vehicles obtained with the new controllable motion model. Steering and acceleration action-primitives were derived by an SGT-traversal in this case. In contrast to the tracking results obtained with XTRACK alone, the situated prediction of movements leads to more realistic expectations about the change in steering angle and velocity. Thus, all four cars can be tracked correctly throughout the whole sequence.

Figure 4 summarizes similar results obtained for a vehicle which approaches a red traffic light where a transporter already stands waiting. While the approaching vehicles stops behind the transporter it gets occluded by a large traffic sign. XTRACK again cannot cope with the change in velocity of the occluded vehicle. Thus, as the actual car reappears from behind the sign, it is already lost by XTRACK. In contrast, by employing SGT-traversal and the action commands following from this traversal, the vehicle is smoothly stopped behind the traffic sign, because the SGT comprises the knowledge that a vehicle usually stops in 
XTRACK alone
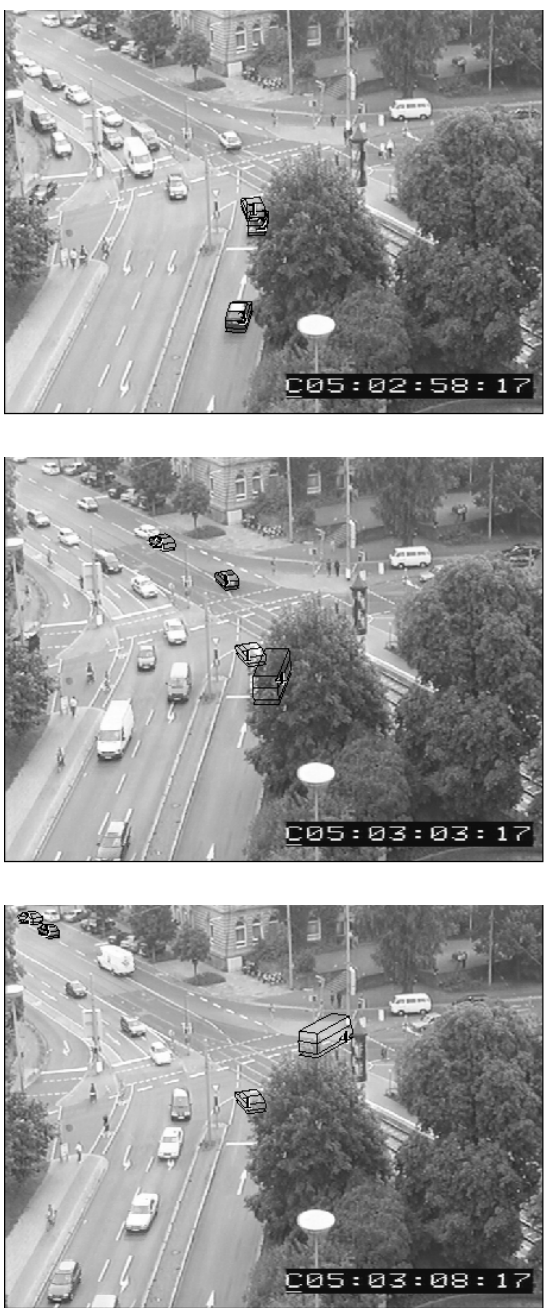

XTRACK with SGT
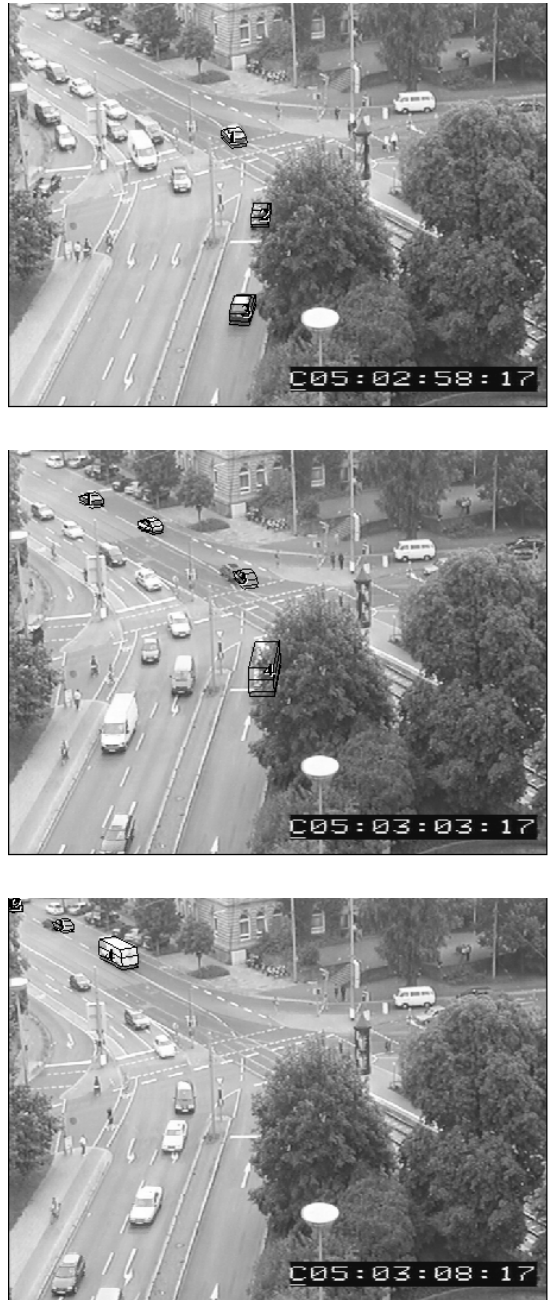

Fig. 3. Representative frames of a video sequence showing an innercity intersection (rows from top to bottom: frames \#1400, \#1650, and \#1900) with superimposed state estimates for four vehicles. The left column shows the tracking results obtained by XTRACK alone. The right column shows tracking results for the same vehicles, but this time with state predictions obtained by SGT-traversal. Note that two vehicles were lost in the left column due to the occluding tree, whereas these vehicles could be tracked successfully with the help of SGT-traversal-based state prediction. 
XTRACK alone
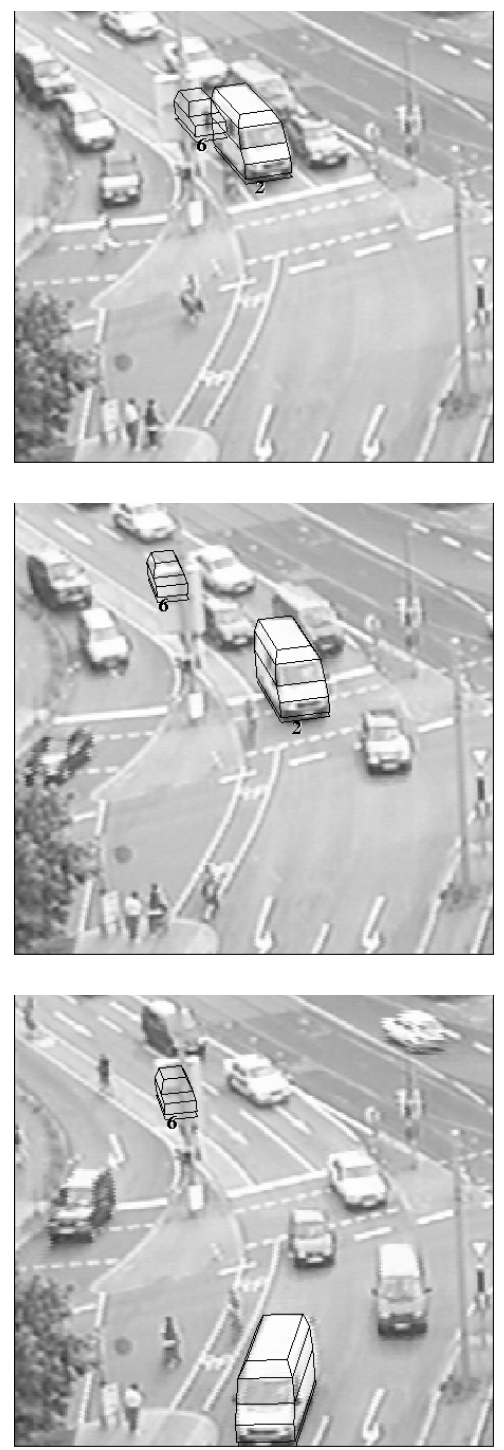

XTRACK with SGT
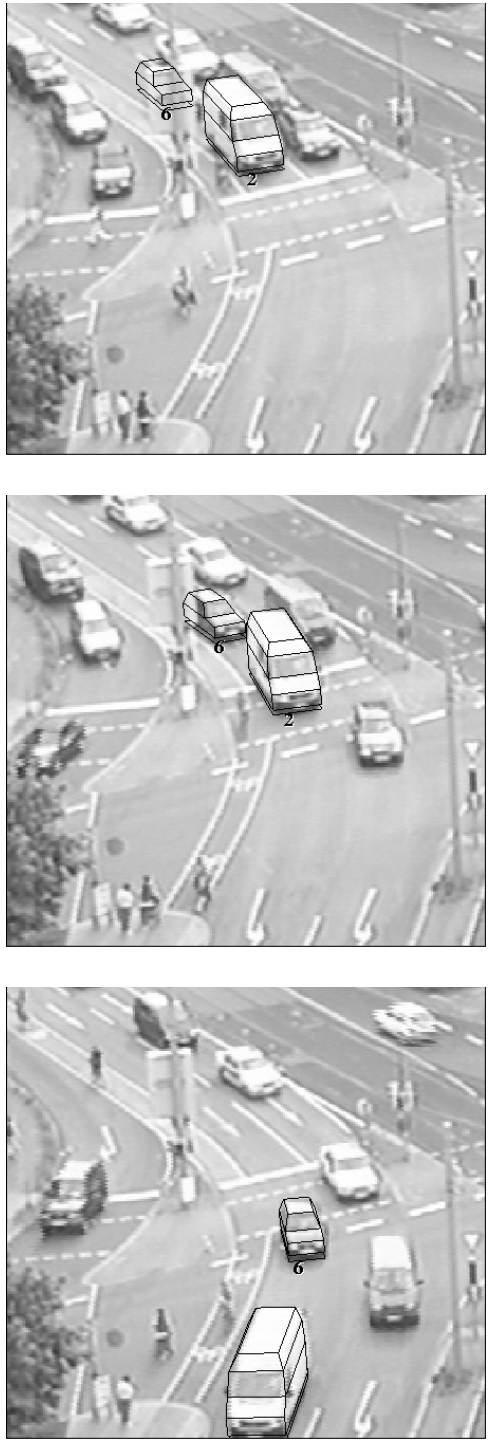

Fig. 4. Clippings from representative frames of the same video sequence as in Figure 3 (rows from top to bottom: frames \#1200, \#1400, and \#1600). The clippings show the tracking results for a vehicle which arrives at a red traffic light, where a waiting transporter is already standing. In the left column, tracking results of XTRACK alone can be seen: here, the vehicle was lost during an occlusion. The right column shows tracking results obtained by XTRACK with the help of SGTs. In this case, the vehicle was tracked correctly. 
front of a stationary obstacle. As the transporter begins to accelerate again, this leads to a change in situation instantiation of the trailing vehicle, too. Therefore, this vehicle is expected to accelerate as the preceding transporter does. As can be seen in Figure 4, this more elaborated assumption about the acceleration of vehicles leads to successful tracking.

\section{Conclusion}

It has been shown that situation graph trees (SGTs), originally introduced as representation formalisms within a cognitive vision system, fulfill two other demands which might be imposed on formalisms capable of representing behavioral knowledge: the generation of synthetic video sequences and the quantitative exploitation of behavioral knowledge in a predictive manner already during the performance of a computer vision task. The former usage of SGTs could be demonstrated by extending work already presented in [1]. Maximized SGTcompatible behaviors can now be visualized in form of a synthesized video sequence by linking these situation sequences to quantitative vehicle states via a controllable motion model. For the latter usage of SGTs, namely the situated prediction of movements during model-based tracking, the controllable motion model was used again. It has been shown here that the quantitative exploitation of conceptual knowledge about the expected behavior leads to better performance of model-based vehicle tracking.

\section{Future Work}

For the investigations presented here, we assumed that the successor situation of the current agent vehicle situation is always uniquely determinable. This is, however, a very strong and often not satisfied assumption. Future work will concentrate on how competing hypotheses about the successor situation can be modelled and tested for compatibility with the model-based tracking results. This requires a fuzzy traversal of SGTs on the one hand and a function which evaluates and compares the fitting of different vehicle states - following from different hypotheses - to the data estimated by state-of-the-art model-based tracking approaches.

\section{A Externally Controlled Vehicle Motion Model}

The state of a vehicle at a time point $k$ shall be given as:

$$
\mathbf{x}_{k}=\left(x_{k}, y_{k}, \theta_{k}, v_{k}, \psi_{k},\right)^{T}
$$

where $x$ and $y$ denote the $\mathrm{X}$ - and $\mathrm{Y}$-position of the reference point of the vehicle. $\theta$ stands for the orientation of the vehicle in the ground plane. $v$ denotes the velocity in the direction of that orientation. $\psi$, finally, represents the steering 


\begin{tabular}{|l|l|}
\hline System Model & $\mathbf{x}_{k}=f\left(\mathbf{x}_{k-1}, \mathbf{u}_{k-1}\right)+\mathbf{w}_{k-1}$, where $\mathbf{w}_{k-1} \sim N(\mathbf{0}, Q)$ \\
\hline Measurement Model & $\mathbf{z}_{k}=h\left(\mathbf{x}_{k}\right)+\mathbf{v}_{k}$, where $\mathbf{v}_{k} \sim N(\mathbf{0}, R)$ \\
\hline \hline Initial Conditions & $E\left[\mathbf{x}_{0}\right]=\hat{\mathbf{x}}_{0}, E\left[\left(\mathbf{x}_{0}-\hat{\mathbf{x}}_{0}\right)\left(\mathbf{x}_{0}-\hat{\mathbf{x}}_{0}\right)^{T}\right]=P_{0}$ \\
\hline Other Assumptions & $E\left[\mathbf{w}_{j} \mathbf{v}_{k}^{T}\right]=0$ for all $j, k$ \\
\hline \hline Estimate Prediction & $\hat{\mathbf{x}}_{k}^{-}=f\left(\hat{\mathbf{x}}_{k-1}^{+}, \mathbf{u}_{k-1}\right)$ \\
\hline Covariance Prediction & $P_{k}^{-}=F_{k}\left(\hat{\mathbf{x}}_{k-1}^{+}\right) P_{k-1}^{+} F^{T}\left(\hat{\mathbf{x}}_{k-1}^{+}\right)+Q$ \\
\hline Kalman Gain Matrix & $K_{k}=P_{k}^{-} H_{k}^{T}\left(\hat{\mathbf{x}}_{k}^{-}\right)\left[H_{k}\left(\hat{\mathbf{x}}_{k}^{-}\right) P_{k}^{-} H_{k}^{T}\left(\hat{\mathbf{x}}_{k}^{-}\right)+R\right]^{-1}$ \\
\hline Estimate Update & $\hat{\mathbf{x}}_{k}^{+}=\hat{\mathbf{x}}_{k}^{-}+K_{k}\left[\mathbf{z}_{k}-h\left(\hat{\mathbf{x}}_{k}^{-}\right)\right]$ \\
\hline Covariance Update & $P_{k}^{+}=\left[I-K_{k} H_{k}\left(\hat{\mathbf{x}}_{k}^{-}\right)\right] P_{k}^{-}$ \\
\hline
\end{tabular}

Table 1. Kalman filter equations according to the notation from [8]. $F$ denotes the Jacobian of the system function $f$, whereas $H$ stands for the Jacobian of the measurement function $h$. Both are needed for linearization of the non-linear system and measurement function, respectively. $\mathbf{x}$ denotes the real system state, while $P$ denotes the error covariance matrix with which this state is estimated.

angle of the vehicle. The movement of a vehicle is assumed to be acceptably modelled as:

$$
\mathbf{x}_{k}=\left(\begin{array}{c}
x_{k-1} \\
y_{k-1} \\
\theta_{k-1} \\
v_{k-1} \\
\psi_{k-1}
\end{array}\right)+\left(\begin{array}{c}
v_{k-1} \Delta t \cos \left(\theta_{k-1}+\psi_{k-1}\right) \\
v_{k-1} \Delta t \sin \left(\theta_{k-1}+\psi_{k-1}\right) \\
\arctan \left(\frac{v_{k-1} \Delta t \sin \left(\psi_{k-1}\right)}{\mathcal{L}}\right) \\
\Delta v_{k} e^{-\Delta t D_{v}} \\
\Delta \psi_{k} e^{-\Delta t D_{\psi}}
\end{array}\right)
$$

where $\Delta t$ is the time increment from time point $k-1$ to time point $k$. With $\mathcal{L}$ we denote the length of the vehicle currently modelled. $\Delta v_{k}$ and $\Delta \psi_{k}$ are the velocity and steering angle increments, respectively, following from external control inputs at time point $k . D_{v}$ and $D_{\psi}$ are parameters defining the damping of changes forced onto the velocity and the steering angle. We assume that given a desired speed value $\bar{v}_{k}$ - the speed increment for time point $k$ is simply defined as $\Delta v_{k}=\bar{v}_{k}-v_{k-1}$. Similarly assuming that a desired orientation of $\bar{\theta}_{k}$ is given, we define the desired steering angle $\bar{\psi}_{k}$ which would lead to such a desired orientation $\bar{\theta}_{k}$ (compare [13]) as:

$$
\bar{\psi}_{k}=\arcsin \left(\frac{\mathcal{L} \tan \left(\bar{\theta}_{k}-\theta_{k-1}\right)}{v_{k-1} \Delta t D_{\psi}}\right) .
$$

Thus, the steering angle increment follows from $\Delta \psi_{k}=\bar{\psi}_{k}-\psi_{k-1}$. We assume the system under observation to be excited by a deterministic control input $\mathbf{u}$ which shall not affect the state measurement. The system function $f$ and measurement function $h$ are assumed to be constant with respect to time. In the same way, we assume $\mathbf{w}$ and $\mathbf{v}$ to be white noise with time-independent covariances. The Kalman filter equations are summarized in Table 1. For details 
about the measurement function see [13]. The Jacobian $F$ of the system function $f$, which is needed to linearize the non-linear system function, follows from

$F=\left(\begin{array}{cccc}10 & -v \Delta t S & \Delta t C & -v \Delta t S \\ 01 & v \Delta t C & \Delta t S & v \Delta t C \\ 0 & 1 & \frac{\Delta t \sin (\psi)}{\mathcal{L}+\frac{v^{2} \Delta t^{2} \sin ^{2}(\psi)}{\mathcal{L}}} & \frac{v \Delta t \cos (\psi)}{\mathcal{L}+\frac{v^{2} \Delta t^{2} \sin ^{2}(\psi)}{\mathcal{L}}} \\ 00 & 1+\left(\frac{\partial \bar{v}}{\partial v}-1\right) e^{-\Delta t D_{v}} & 0 \\ 00-\frac{\mathcal{L} e^{-\Delta t D \psi}}{v \Delta t \cos ^{2}(\bar{\theta}-\theta) \sqrt{1-\frac{\mathcal{L}^{2} T^{2}}{v^{2} \Delta t^{2} D_{\psi}^{2}}}}-\frac{\mathcal{L} T e^{-\Delta t D_{\psi}}}{\Delta t D_{\psi} \sqrt{1-\frac{\mathcal{L}^{2} T^{2}}{v^{2} \Delta t D_{\psi}}}} & \\ 0 & \left.-\frac{\partial \bar{\psi}}{\partial \psi}-1\right) e^{-\Delta t D_{\psi}}\end{array}\right)$

with the abbreviations $S=\sin (\theta+\psi), C=\cos (\theta+\psi)$, and $T=\tan (\bar{\theta}-\theta)$.

\section{Acknowledgements}

The authors gratefully acknowledge support for this work by the European Union under contract No. IST-2000-29404 (CogViSys).

\section{References}

1. M. Arens and H.-H. Nagel: Behavioral Knowledge Representation for the Understanding and Creation of Video Sequences. In: A. Günter, R. Kruse, and B. Neumann (Eds.): Proc. of the 26th German Conf. on Artificial Intelligence (KI-2003), 15-18 September 2003, Hamburg, Germany, (LNAI) 2821, Springer-Verlag, Berlin u.a. 2003, pp. 149-163.

2. M. Arens, A. Ottlik, and H.-H. Nagel: Natural Language Texts for a Cognitive Vision System. In: F. van Harmelen (Ed.): Proc. of the 15th European Conf. on Artificial Intelligence (ECAI-2002), 21-26 July 2002, Lyon, France, IOS Press, Amsterdam 2002, pp. 455-459.

3. A. Blocher and J. R. J. Schirra: Optional Deep Case Filling and Focus Control with Mental Images: ANTLIMA-KOREF. In: C. S. Mellish (Ed.): Proc. of the 14th Int. Joint Conf. on Artificial Intelligence (IJCAI-95), 20-25 August 1995, Montréal, Canada, Morgan Kaufmann, San Mateo, CA, USA 1995, pp. 417-423.

4. A. Egges, A. Nijholt, and P. Nugues: Generating a 3D Simulation of a Car Accident from a Formal Description: the CarSim System. In: V. Giagourta and M. G. Strintzis (Eds.): Proc. of the Int. Conf. on Augmented, Virtual Environments and Three-Dimensional Imaging (ICAV3D), 30 May - 1 June 2001, Mykonos, Greece, pp. $220-223$.

5. A. Gelb (Ed.): Applied Optimal Estimation. The MIT Press, Cambridge/MA, USA, London/UK, 1974. 
6. R. Gerber: Natürlichsprachliche Beschreibung von Straßenverkehrsszenen durch Bildfolgenauswertung. Dissertation, Fakultät für Informatik, Universität Karlsruhe (TH), Januar 2000 (in German); see: http://www.ubka.uni-karlsruhe.de/cgibin/psview?document $=2000 /$ informatik/8.

7. R. Gerber and H.-H. Nagel: Occurrence Extraction from Image Sequences of Road Traffic Scenes. In: L. van Gool and B. Schiele (Eds.): Proc. of the Workshop on Cognitive Vision, 19-20 September 2002, ETH Zurich, Switzerland; pp. 1-8, see: http://www.vision.ethz.ch/cogvis02/finalpapers/gerber.pdf.

8. M. S. Grewal and A. P. Andrews: Kalman Filtering: Theory and Practice. Prentice Hall, Englewood Cliffs, NJ, USA, 1993.

9. M. Haag und H.-H. Nagel: 'Begriffliche Rückkopplung' zur Behandlung temporärer Verdeckungssituationen in der Bildfolgenauswertung von Strassenverkehrsszenen. In: J. Dassow und R. Kruse (Hrsg.): Informatik'98, Informatik zwischen Bild und Sprache, 28. Jahrestagung der GI, 21-25 September 1998, Magdeburg, Deutschland, Informatik aktuell, Springer-Verlag, Berlin u.a. 1998, pp. 13-22 (in German).

10. M. Haag and H.-H. Nagel: Combination of Edge Element and Optical Flow Estimates for 3D-Model-Based Vehicle Tracking in Traffic Image Sequences. International Journal of Computer Vision 35:3(1999) 295-319.

11. M. Haag and H.-H. Nagel: Incremental Recognition of Traffic Situations from Video Image Sequences. Image and Vision Computing 18:2 (2000) 137-153.

12. R. J. Howarth and H. Buxton: Conceptual Descriptions from Monitoring and Watching Image Sequences. Image and Vision Computing 18:2 (2000) 105-135.

13. H. Leuck: Untersuchungen zu einer systematischen Leistungssteigerung in der modellbasierten Bildfolgenauswertung. Dissertation, Fakultät für Informatik, Universität Karlsruhe (TH), Juli 2000; Berichte aus der Informatik; Shaker-Verlag Aachen 2001 (in German).

14. A. Mukerjee, K. Gupta, S. Nautiyal, M.P. Singh, and N. Mishra: Conceptual Description of Visual Scenes from Linguistic Models. Image and Vision Computing 18:2 (2000) 173-187.

15. H.-H. Nagel: From Image Sequences towards Conceptual Descriptions. Image and Vision Computing 6:2 (1988) 59-74.

16. H. H. Nagel, M. Haag, V. Jeyakumar, and A. Mukerjee: Visualisation of Conceptual Descriptions Derived from Image Sequences. 21st DAGM-Symposium, 15-17 September 1999, Bonn, Springer-Verlag, Berlin u.a 1999, pp. 364-371.

17. B. Neumann: Natural Language Description of Time-Varying Scenes. In D. Waltz (Ed.): Semantic Structures: Advances in Natural Language Processing, Lawrence Erlbaum Publishers, Hillsdale/NJ · Hove and London/UK 1989, pp. 167-206.

18. K. H. Schäfer: Unscharfe zeitlogische Modellierung von Situationen und Handlungen in Bildfolgenauswertung und Robotik. Dissertation, Fakultät für Informatik, Universität Karlsruhe (TH), Juli 1996; Dissertationen zur Künstlichen Intelligenz (DISKI) 135; infix-Verlag: Sankt Augustin 1996 (in German).

19. J. R. J. Schirra: Bildbeschreibung als Verbindung von visuellem und sprachlichem Raum. Dissertation, Fakultät für Informatik, Universität des Saarlandes, Saarbrücken, April 1994; Dissertationen zur Künstlichen Intelligenz (DISKI) 71; infix-Verlag: Sankt Augustin 1994 (in German).

20. V. T. Vu, F. Brémond, and M. Thonnat: Human Behaviour Visualisation and Simulation for Automatic Video Understanding. In V. Skala (Ed.): Proc. of the 10th Int. Conf. in Central Europe on Computer Graphics, Visualization and Computer Vision (WSCG-2002), Plzen-Bory, Czech Republic, 2002; see Journal of WSCG 10:2 (2002) 485-492, ISSN 1213-6972. 